\title{
A Bidder Behavior Learning Intelligent System for Trust Measurement
}

\author{
El-Sayed Towfek El-kenawy \\ Department of Computer and \\ Systems Engineering, \\ Faculty of Engineering, \\ Mansoura University, Egypt
}

\author{
Ali Ibraheem El-Desoky \\ Department of Computer and \\ Systems Engineering, \\ Faculty of Engineering, \\ Mansoura University, Egypt
}

\author{
Amany M. Sarhan \\ Department of Computer scienecs, \\ Faculty of Computer and \\ Information, \\ Tanta University, Egypt
}

\begin{abstract}
With the spread of e-commerce fields over the world especially an online auctions segment, the problems of internet crimes increased in the last years. The online auctions face a serious problem of trust among the participants where users have no information about the others, and have no relations among them except the commercial transactions; this allows fraud to occur by malicious users. We proposed a framework depend on "Bidder Behavior" consist of three algorithms the first " Bidder Increase Price Behavior"(BIPB), the second "Search In Stored Data Base"(SSDB) and the third "Person Recursive Search" (PRS) to improve the performance of trust measurement
\end{abstract}

\section{Keywords}

Bidder Increase Price Behavior (BIPB), Search In Stored Data Base (SSDB), Person Recursive Search (PRS) ,Online Auctions, Learning Intelligent System, Negotiation; Reputation; Trust, E-fraud.

\section{INTRODUCTION}

An e-auction is an indirect sale transaction type of ecommerce. An e-auction increases competition among vendors in addition to broaden the set of potential consumer. In an auction (electronic or not), buyers compete to get the right to buy the product. There are many types of auctions such as an English auction in which the highest price wins, the bidding price increase until no more did happen, Dutch auction in which the bidding process start with a top price decreasing until an auction participant accepts the price stated, American Auction or Closed Bid Auction in which the highest bid wins, when the bid envelopes are opened and Vickrey Auction or Uniform Second Price in which the winner pays the second highest price and not his own offer. [1]

The main stages of sale transaction according to the Consumer Buying Behavior:
1. Identification.
2. Product Brokering.
3. Merchant Brokering.
4. Negotiation.
5. Final Sale.
6. Evaluation.

The online auction has many benefits such as:

1. substantial cost savings through auction being online and instantaneous;
2. Reducing the sourcing cycle time.

3. The ability to open up procurement to a wider market allowing suppliers to make bids without being restricted by geographic location.

4. An efficient, open and transparent system and clear audit trail of transactions.

5. Greater insight of buyers into the dynamics of the marketplace and an indication of the true market price for goods and services.

6. Anonymous online event creates a "level playing field" i.e. a fair and competitive market.

7. Suppliers can, of course, use E-Auction to arrive at market prices for their own purchases of raw materials and components.[2]

The participants during the previous sale stages don't know each other, this makes the online auction as a suitable environment for internet crimes like fraud, which can occur in two ways one by Bid Shilling in which seller create false identities to push up the bid price, and Bid Shielding in which the other bidder is discouraged from the participants of online auction. This is explicit in the following chart as a statistic described the Percentage of complaint categories in the reported cases of cyber crime on the IC3 platform in the U.S. in 2010.[2]

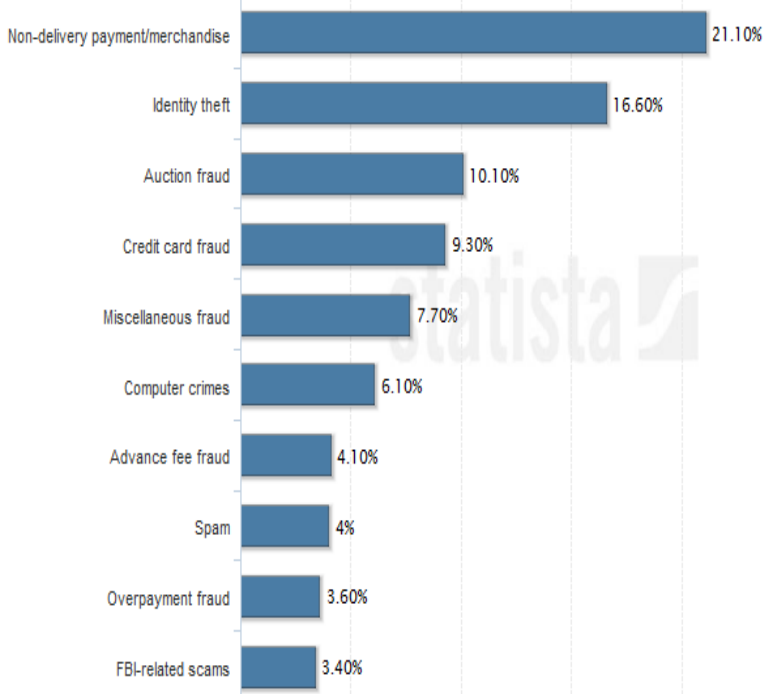

Figure 1.IC3 chart for internet crimes 
The National White Collar Crime Center [3] declared that the exploration of the eBay auction system is also significant for the experiment carried out since eBay fraud accounts for more than half of online auction fraud. IC3 declared in the (Federal Bureau of Investigation) FBI report about fraud 2012 high rate of complaints in auto fraud as a case study [3]. It show the percentage complaints by age

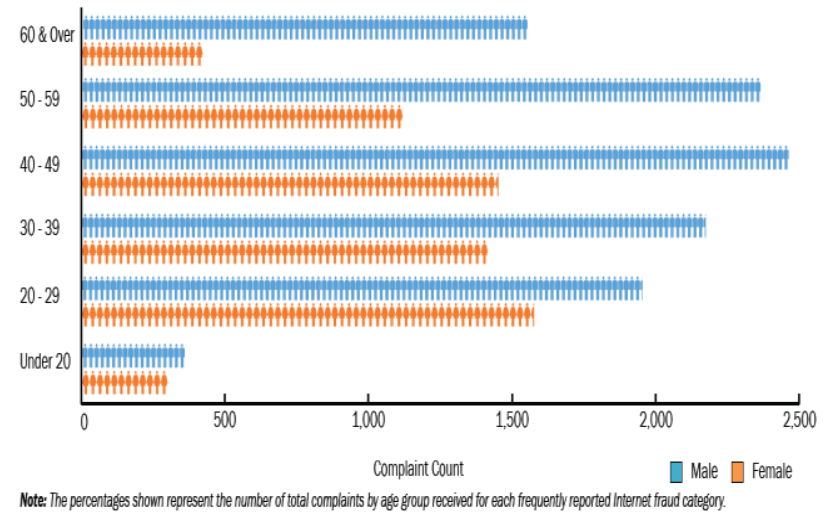

Figure 2.IC3 chart for internet crimes2012

The pervious statics show fraud in cyber crime, Internet users' complaint and how it causes a great loss for all participants'. The fraud can occur through negotiation stage.

Negotiation is a Process by which group of software agents communicate with each other to reach a mutually acceptable agreement on any matter. [4].

There are two major issues of trust for the users occur in emarketplaces:

1. The potential buyer can't access physically to the product, so the seller could misrepresent his service or product quality to ask for a higher price.

2. The seller or the buyer may not execute the agreement reached at the e-marketplace asking later to renegotiate or even refuse to commit the transaction.

The e-auction processes take place using the agent technology which is used to avoid the problem of continuous monitoring process during the auction. Learning intelligent system are software agents assist participants in the auctions by automating some of the key aspects of the process. Auction aggregators use intelligent agents to find and track auctions on multiple auction sites. The agents in e-negotiation dialogs based on their own requirements until reaching an agreement on one or multi issues of the negotiation. Using heuristic algorithms to develop negotiation agents ' decision making mechanism is useful. [5]

\section{REPUTATION AND TRUST IN E- AUCTION}

The participant of online auction is unaware of the details of the seller; they have no other option except trust the feedback mechanism [5], so there is a gap between the auction participants depending on the trust and participant's reputation. There is a difference between trust and reputation, reputation is a quantity relative to the particular embedded social network of evaluating agent and encounter history, trust is a dyadic quantity between the trustee and the trustier which can be obtained from the reputation data about trustee [6].
Reputation is very important and useful in multi-agent systems because there are large numbers of agents interacting during the auction. For example reputation can be seen as follows: allow buyers to choose the best seller in the auction system, so the reputation motivates seller to do the best to avoid bad reputation.

The field lines of reputation: [7]

1. Define the trustworthiness of an agent using relationships between participants.

2. Collect information from the aggregation of rating retrieval from the communication between members.

3. Describe the trustworthiness of an agent truly.

Summary of Critical success factors of $\mathrm{C} 2 \mathrm{C}$ auction about security and trust of e-auction privacy (PRI) that include how users' personal data is used by eauction, protection (PRO) to prevent, avoid, remove any malicious action, Detection (DET) by using software like firewalls, virus prevention to detect any unauthorized users, Reputation (T), the website's reputation is associated to the size, how long the online auction has been working and the other organization deal with the auction website, Multi agent system (MAS) is a software has the ability of detecting, scheduling, development , planning and decision making.[8]

The positive reputation rating is not the only factor to increase the bidder's probability of winning the auction, there is a great role of communication and reputation in e-auction, messages can be used to reduce the asymmetric information associated with the transaction. [7]. the feedback ratings from the participants of the online auction are unreliable and limited.

Trust and reputation management plays an efficient role in collaboration among network participant that might not have sufficient prior knowledge about each other. Reputation based trust is often employed to help identify and avoid malicious players and protect a networked computing system from possible abuses. There are many challenges in trust and reputation management for future internet computing application as trust and reputation representation.

Trust and reputation have a central role in interaction in multiagent systems (MAS) in which agents can work freely, can enter and leave the system at any time. The individual components act in an autonomous and flexible manner in MAS to achieve their objectives. The agents' features in MAS can be considered as:

1. The agents' are an unreliable and self interested.

2. The agents have no details about their environment.

3. No central authority can control all the agents.

Despite these uncertainty features the interaction between agents has to take place. There are four different types of trust and reputation that are used to get the best reliability for system reputation, Interaction trust which is calculated from direct interaction and past experience. Role based trust is defined by the relationships between the agents. Witness reputation contains the reports of witnesses about the agent behavior. Certificate reputation present references about the agent behavior provided by the other agents. [9]

The important factors that affect on e-auction trust and reliability: 
- Credibility: professional appearance of the auction site, up to date information, good interaction...

- Security: high security issues must be applied.

- Added Incentives: giving important information regarding the offers available.

- Experience: feedback facilities should be applied to share past experiences which play an important role in trust management.

Trust is built in negotiation process which need a management that feature a policy language based on state machines. It is supported by Lifecycle management and automated runtime enforcement tools. A trust negotiation system used to avoid some problems as scalability problems when attempting to store identifying information for each request, where web service providers don't know requester identities. The interaction occurs between service requester and service provider, the both own credential that used to negotiate trust. [10]

\section{RELATED WORK}

The fraud during e-auction processes reflects bad reputation for this auction and its participants, so thus reduce the demand for these online auctions. Many studies discussed how to protect the participants from fraud using many techniques, one uses trust models to calculate each participant trust, other one uses special comparable algorithms to predict that the auction users are trusted or un trusted, and some auctions may use a third party like payment services as escrow services. These related works discuss these techniques.

\subsection{The third party services}

To protect the consumers from fraud, proposed services like escrow services are used to help users how to buy and sell online without getting scammed. The problem: how to exchange money for an online auction sale like on (E-bay) without getting scammed. If you are selling something, what is an acceptable form of payment when you can't take a credit card? If you take a check and ship the product, by the time the check bounces, the merchandise is gone! Escrow.com, an accredited escrow company, acts as a secure third party to protect the Buyer and Seller.

\subsection{Predicting the participants' behavior using feedback features}

A study in 2004 explained the impact of product classification for online auctions and how it affects the negative feedback. The product classified to four features "product description complexity, sensory products, variable functionality, and fragility "which affect consumer complaints. [10]

At 2005 the study of [11] presented two algorithms for reputation depending on data mining to analyze the topology of seller-buyer connection to derive useful knowledge about the participants using their credibility and density. One compute the credibility of the participants by an iterative search of the connection between the inter-participants, the other one discover clusters of participants who are densely connected through committed auction.

In 2005 a study in [12] proposed a comparable algorithm to discover negative feedback through feedback comments where the online auctions are biased to positive feedback and neglect the negative feedback, no auction site support personalize trust value. For example e-Bay trust can be gained using feedback comments but some problems appear as, the user can choose not to display feedback comments, or unpaid items the buyers are not allowed to write a rating comment. This explains the negative feedback.

A new framework for agent based trust management in online auction was proposed in 2008 in the paper [13] to facilitate real time trust re-evaluation by updating user roles and access permissions dynamically, where the reputation models depend on prior history of users and/or feedback from the other entities, and there is no effective mechanism to prevent users from giving false information when making a recommendation.

A new method for mining reputation was presented in 2009 in [14] that access the reliability and questionability of seller for negative and positive reputation by constructing S-graph which reflects the social linkage between seller and buyer, that identifying missing feedback that detect misbehaving contractor and counteract fraud.

The study of [15] at 2010 used collection channels which refer to how information collected from evaluators, the information can be collected through three formats:

1. Rating scales in which evaluator's rate the product from 1:5.

2. Text comments in which evaluators are asked to write a comment.

3. Other formats like videos and pictures.

\subsection{Trusted models using intelligent agent}

The other studies discuss proposed trusted models that calculate the participants trust using intelligent agent technology as follows. Spares is a simple reputation mechanism can be implemented irrespective the number of ratings. There are basic principles provided by spore's model services:

1. The user reputation is built during its activity starting with minimum reputation value.

2. No matter how unreliable the user is the reputation value of a user doesn't fall below the reputation of a new user.

3. The reputation value of the user is updated based on the feedback provided by the others to reflect the last trustworthiness.

4. The system keeps the most recently submitted rating in the case of two users may interact more than once.

5. Smaller rating change happened with the users with high reputation value.

Histos is a complex reputation mechanism that assumes that there are many rated interactions to create a dense web of pairwise ratings. It used to overcome the problem of connecting among the users by building a more personalized system in Weaving a Web of Trust, that there is connected path between two users. [2002][16]

The model of 2005 named as Fire: An Integrated Trust and Reputation Model for Open Multi-Agent Systems, This model is suitable in MAS in which each agent is responsible for evaluating and storing its trust information, can produce an associated reliability and trust measure. This model combines between the four different types of reputation and trust: 
1. Interaction trust: calculated from direct interaction and past experience.

2. Role based trust: defined by the relationships between the agents.

3. Witness reputation: contain the reports of witnesses about the agent behavior.

4. Certificate reputation: references about the agent behavior provided by the other agents. [17]

In 2006 A new quantitative trust model for negotiation agents using argumentation was proposed to provide a secure environment for agent negotiation using multi agent systems. The centralized mechanisms such as digital certificate are difficult to be attached because there is no check on the system, if the trusted authorities are compromised, and not suitable for argumentation. Multi-agent systems, protocols used to achieve a meaningful interaction between agents. Such protocols describe allowed communicative acts between agents, the flexibility protocols can be achieved by combining small dialogue games using logical rules about the reasoning agent which can specify this combination. The framework must allow one entity to assume that the second entity will behave as the first entity expect exactly. This model is an efficient and gathers the most important factors:

1. The trustworthiness of confidential agents;

2. The target's trustworthiness according to the point of view of confidence agents
3. The number of interactions between confidence agents and the target agent;

4. The timely relevance of information transmitted by confidence agents.

Another model was proposed in 2009 called Agent Based Trust Management Model Based on Weight Value Model for Online Auction. This model is used to improve the performance and scalability of e-auctions by providing efficient trust management methodology using agent technology to avoid the problem of monitoring the proceeding of the auction taking into account some consideration factors such as the time taken for the auction and the original price obtained versus the price expected. [18]

\section{THE PROPOSED FRAMEWORK}

We propose a framework consists of three algorithms the first "Bidder Increase Price Behavior" (BIPB), the second "Search in Stored Data Base" (SSDB) and the third "Person Recursive Search" (PRS) to improve the performance of trust measurement using learning intelligent system to predict the other participants' behavior.

\subsection{Increase Price Behavior (BIPB)}

The BIPB algorithm will analysis the winners' behavior in increasing price and stored by the other trusted users from the other auction sites then comparing participants' behavior, taking into account the category, the rarity of the item and time level. As shown in figure (3)

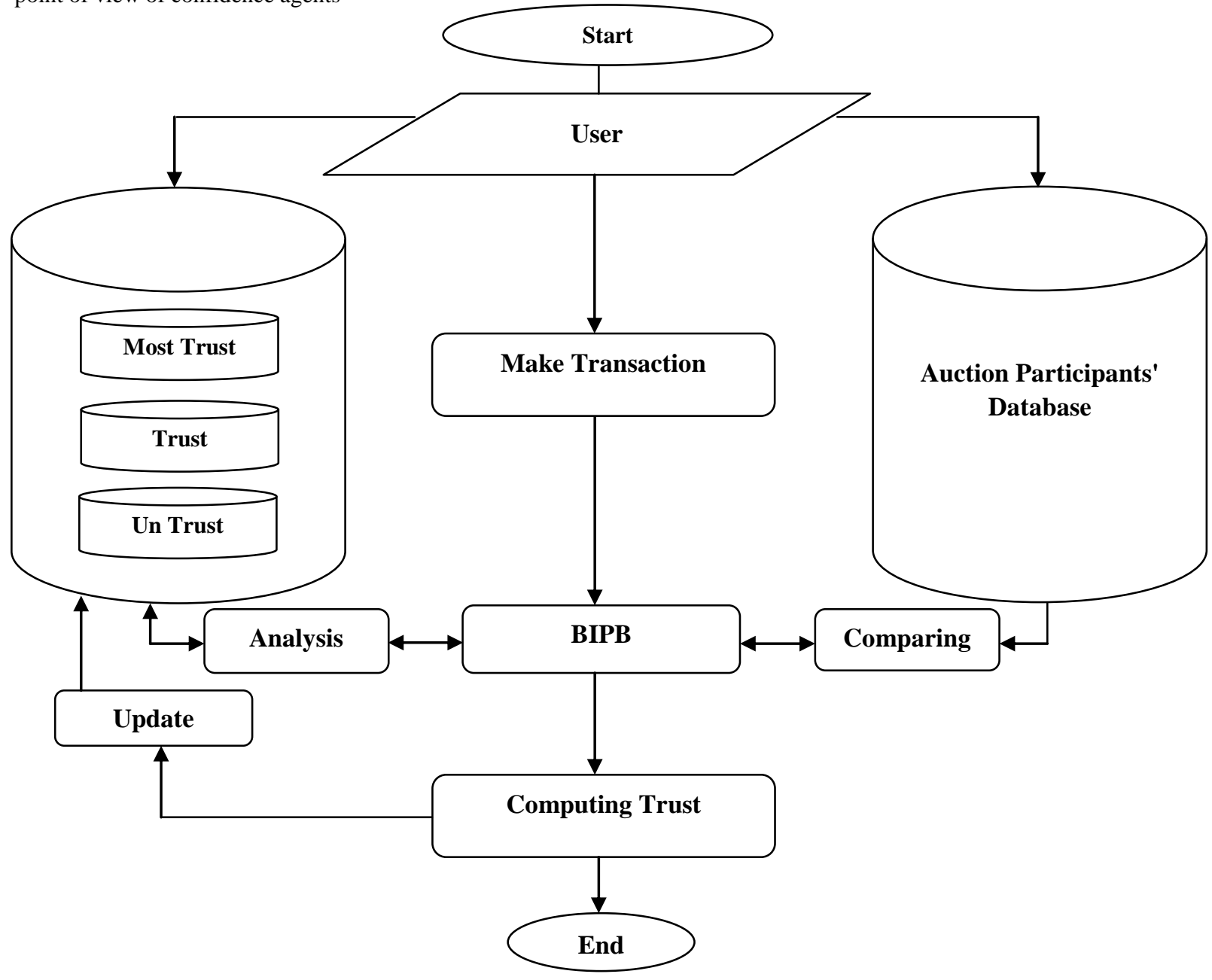

Figure (3) BIPD flow chart 


\subsection{Search In Stored Data Base (SSDB)}

The SSDB algorithm receives the information about each participant, this information divided to the user profile and historical transactions for this user, which is stored in the existing database, then the SSDB, compare this information with a stored trusted database that stored by other trusted users from other auction sites. This trusted data consists of measures for testing trusted users. These measures are based on the features of trusted users which are collected from trusted users' profiles, their transactions and feedbacks. After mining the information using SSDB algorithms and applying decision making process. As shown in figure (4).

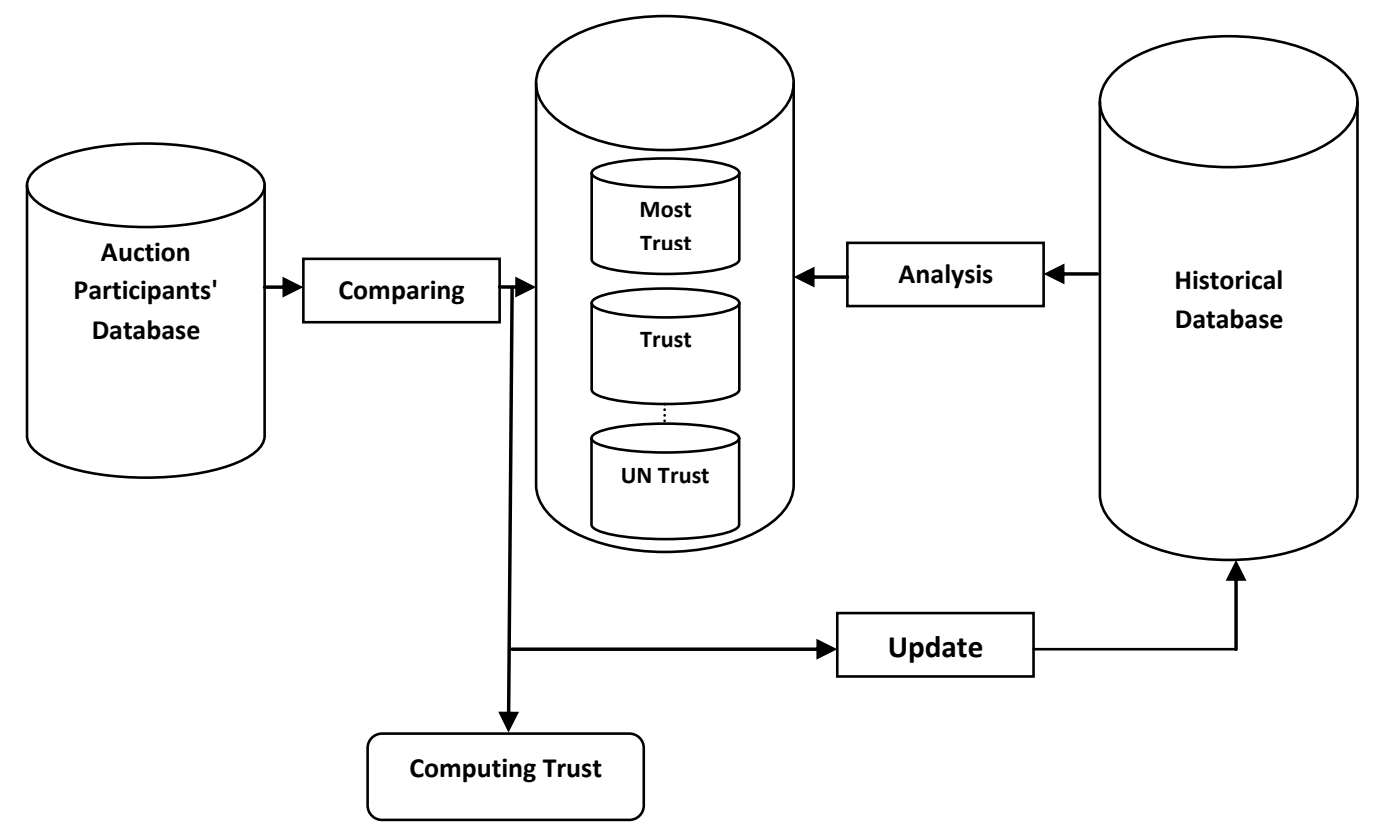

Figure (4) SSDB flow chart

\subsection{Person Recursive Search (PRS)}

The APA algorithm described a trust propagation mechanism which uses the output of the auction rule to generate personalized trust between person's transactions as a social network graph which edges length and thickness as a function of strength and goodness. Taking into account the location, face book friend, time of relation and the category. As shown in the figure (5)

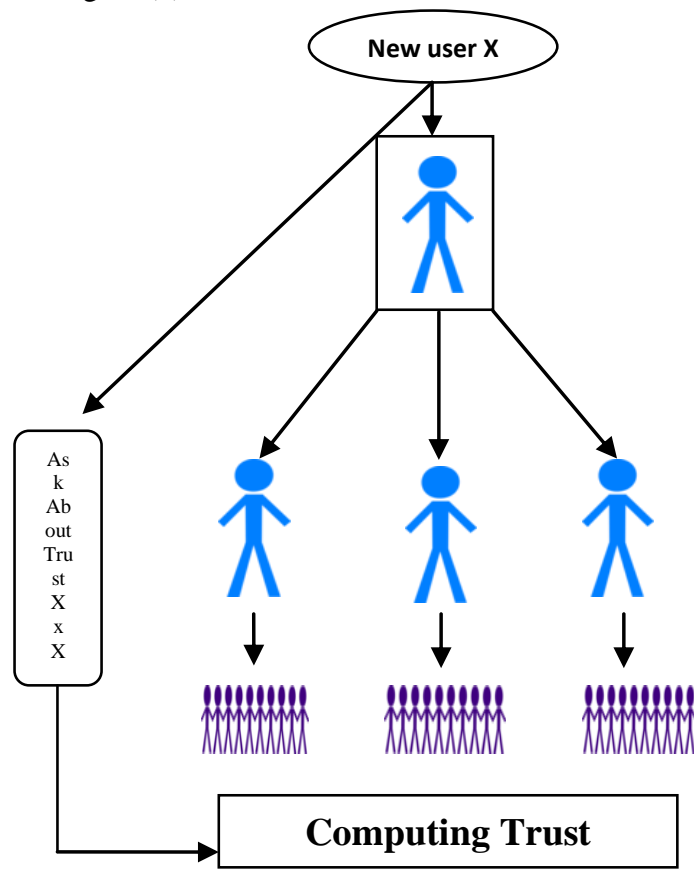

Figure (5) Search about persons may know $X$
Using technology Ask Person About Person Recursive Search ( PRS) that allow users to directly make their recommended social network, extracting trust from feedback comments applying classification algorithms to capture negativity in feedback comments. As shown in figure (6)

Function Find friends know (person, $\mathrm{X}$ )

// order friends of person knowing $\mathrm{X}$ by the location, facebook friends, time of relation and the category.

End Function

Function Compute trust (person)

If person knows $\mathrm{X}$

Voters $=$ Voters +1

Trust $(\mathrm{X})=[\operatorname{Trust}(\mathrm{X})+($ Trust $($ person $) *$ Trust $($ person, $\mathrm{X}))] / \mathrm{Nub}$ of Voters

End If

For each Find friends know (person, $\mathrm{X}$ ) as (J)

Compute trust (J)

End for each

Return Trust (X)

End Function

For each Friends ( $\mathrm{x}$ ) as person

// Friends ...People may know (X)

Trust $(\mathrm{X})=$ Compute Trust (person)

End for each

Print Trust $(\mathrm{X})$

Figure (6) Recursive Search function 


\section{RESULTS}

By running the algorithms, we can get a lot of results we can see some of it. For example

1. For every bidder relation between number of bids and category. As shown in figure(7)

\begin{tabular}{|l|l|}
\hline $\begin{array}{c}\text { Category(Electronics) } \\
\text { Item }\end{array}$ & Number of bids \\
\hline Laptop & 90 \\
\hline Mobile & 75 \\
\hline I Pad & 35 \\
\hline
\end{tabular}

Figure (7) Relation between number of bids and category

2. For every bidder relation between number of bids and time. As shown in figure(8)

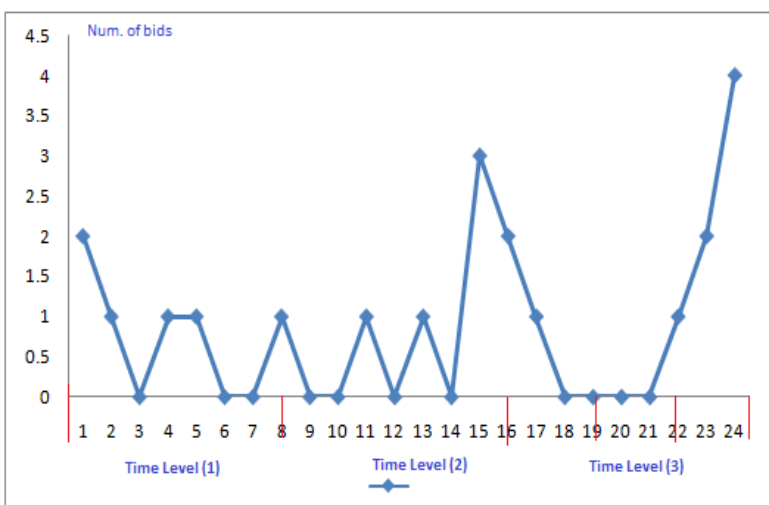

Figure (8) Relation between number of bids and time

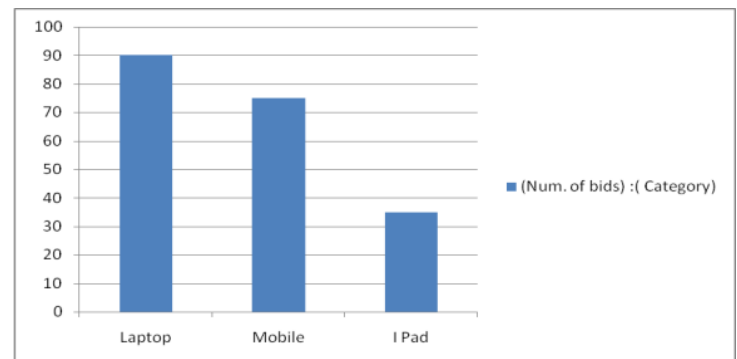

We also can have all relation and behavior, these stored in data base finally the algorithm will analysis it then predict and computing trust. As shown in table (1)

Table (1) Bidder trust status

\begin{tabular}{|c|l|l|l|l|l|}
\hline $\begin{array}{c}\text { Bid } \\
\text { der } \\
\text { Na } \\
\text { me }\end{array}$ & $\begin{array}{l}\text { (BI } \\
\text { PB } \\
\text { usr } \\
\text { ust }\end{array}$ & $\begin{array}{l}\text { (SS } \\
\text { DB }) \\
\text { Tru } \\
\text { st }\end{array}$ & $\begin{array}{l}\text { (PR } \\
\text { S)T } \\
\text { rust }\end{array}$ & $\begin{array}{l}\text { Total } \\
\text { Trust\% }\end{array}$ & Bidder status \\
\hline A & & & & $\begin{array}{c}(75: 100) \\
\%\end{array}$ & Most Trusted \\
\hline B & & & & $\begin{array}{c}(50: 75( \\
\%\end{array}$ & Trusted \\
\hline C & & & & $\begin{array}{c}(35: 50( \\
\%\end{array}$ & Normal \\
\hline D & & & & $\begin{array}{c}(20: 35( \\
\%\end{array}$ & Un Trusted \\
\hline E & & & & $(0: 20(\%$ & Shilling \\
\hline
\end{tabular}

\section{CONCLUSION}

In this paper we have discussed different techniques of reputation and trust systems with online auctions. After surveying the previous studies about detecting the trust and reputation of online auction users. It is important that the user of e-auction be trusted so, we should find a way that detects the user ' status if he is trusted or not depending on his historical data, feedbacks, transactions behavior and ask persons may know him. To reduce the fraud in e-auction, we propose this model using learning intelligent system technology that can predict the users' behavior by analyzing and comparing the users' data with stored trusted data. This facilitates to reduce the fraud that can be occurred by malicious users.

\section{REFERENCES}

[1] Ana Cristina Bicharra Garcia*, Anderson Lopes*, Cristiana Bentes, Electronic Auction with autonomous intelligent agents: Finding opportunities by being there. Inteligencia Artificial, Revista Iberoamericana de Inteligencia Artificial. No.13 (2001), pp. 45-52. ISSN: 1137-3601. (c) AEPIA http://www.aepia.dsic.upv.es/).

[2] The Ministry of Finance, Investments Division report about eAUCTION: THE eWAY OF BUSINESS PROCUREMENT.

[3] 2012_IC3Report.2012.

[4] Azeem Aleem, Albert Antwi-Boasiako, Internet auction fraud: The evolving nature of online auctions criminality and the mitigating framework to address the threat .www. science direct.com International Journal of Law, Crime and Justice 39 (2011)

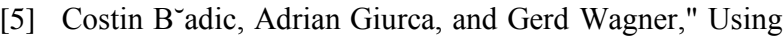
Rules and R2ML for Modeling Negotiation Mechanisms in E-Commerce Agent Systems",2008.

[6] E.Sathiyamoorthy, N.Ch.Sriman Narayana Iyenger \& V.Ramachandran,"Agent Based Trust Management Model Based on Weight Value Model for Online Auctions " International Journal of Network Security \& Its Applications (IJNSA), October 2009

[7] Lik Mui, Mojdeh Mohtashemi, Ari halberstadt, " A Computational Model of Trust and Reputation ", 2002

[8] Hany Abdelghaffar INVISTEGATING THE CRITICAL SUCCESS FACTORS FOR ONLINE C2C AUCTIONS IN EGYPT 2012, European, Mediterranean \& Middle Eastern Conference on Information Systems 2012 (EMCIS2012) June 7-8, Munich, Germany

[9] Shashank Pandit, Duen Horng Chau, Samuel Wang, Christos Faloutsos, " NetProbe: A Fast and Scalable System for Fraud Detection in Online Auction Networks" , 2007

[10] T. Dong Huynh and Nicholas R. Jennings and Nigel R. Shadbolt "FIRE: An Integrated Trust and Reputation Model for Open Multi-Agent Systems"2005

[11] Judy E. Scott , Daw n G. Gregg ," The Impact of Product Classification for Online Auctions",2010.

[12] Mikolaj Morzy, New Algorithms for Mining the Reputation of Participants of Online Auctions, X. Deng and Y. Ye (Eds.): WINE 2005, LNCS 3828, pp. 112121, 2005,Springer-Verlag Berlin Heidelberg 2005 
[13] Haiping Xu, Sol M. Shatz, and Christopher K. Bates." A Framework for Agent-Based Trust Management in Online Auctions",Fift h Internat ional Conference on Information Technology: New Generations.2008.

[14] Mikołaj Morzy, Adam Wierzbickiand Apostolos N. Papadopoulos," Mining online auc t ion social networks for reputation and recommendation"2009

[15] Ling Liu, Malcolm Munro, William Song," EVALUATION OF COLLECTING REVIEWS IN CENTRALIZED ONLINE REPUTATION SYSTEMS".2010
[16] E.Sathiyamoorthy, N.Ch.Sriman Narayana Iyenger \& V.Ramachandran,"Agent Based Trust Management Model Based on Weight Value Model for Online Auctions " International Journal of Network Security \& Its Applications (IJNSA), October 2009

[17] Shashank Pandit, Duen Horng Chau, Samuel Wang, Christos Faloutsos, " NetProbe: A Fast and Scalable System for Fraud Detection in Online Auction Networks", 2007.

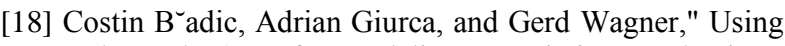
Rules and R2ML for Modeling Negotiation Mechanisms in E-Commerce Agent Systems", 2008 